\title{
The Role of Epicardial Fat Tissue at Cardiovascular Risk Assessment in Hemodialysis Patients with Nutritional Disorder
}

\author{
Mustafa Demir ${ }^{1 *}$, Omer Canpolat ${ }^{2}$, Ayse Das Cerci ${ }^{3}$ and Ayhan Dogukan ${ }^{1}$ \\ ${ }^{1}$ Division of Nephrology, Fırat University School of Medicine, Elazig, Turkey \\ ${ }^{2}$ Department of Emergency Medicine, Elazig Training and Research Hospital, Elazig, Turkey
}

${ }^{3}$ Department of Emergency Medicine, Osmaniye State Hospital, Osmaniye, Turkey

\begin{abstract}
Objective: Protein-energy malnutrition (PEM) is one of the most important risk factors in terms of morbidity and mortality in patients with end-stage renal disease (ESRD) receiving hemodialysis (HD). Therefore, in this population it is important to evaluate the nutritional status and body composition correctly. Our aim was to compare epicardial adipose
\end{abstract} tissue thickness (EAT) in HD patients with and without malnutrition.

Methods: Fifty-six patients were included in the study who were receiving HD therapy for ESRD. Mini Nutritional Assessment (MNA) was administered to determine the nutritional status of the patients. According to MNA scores; patients were divided into two groups as PEM+PEM risk group (group 1, $\mathrm{n}=25$, score $<24$ ) and group with well-nourished (group 2, $n=31$, score $\geq 24$ ). In addition, Tanita SC 330, a body composition analyzer, was used to evaluate the body composition of patients. Transthoracic echocardiography was performed to determine EAT.

Results: Of the 56 patients included in the study, 31 were male and 25 were female. EAT values were significantly different between the two groups $(p=0.032)$. EAT value was higher in Group 2 than in Group $1(p=0.032)$. Phosphor $(P)$ $(p=0.01)$ and $\operatorname{CAXP}(p=0.02)$ values were significantly higher in Group 2 . In addition, fat mass $(p=0.011)$, visceral fat percentage $(p<0.001)$, muscle mass $(p<0.001)$, metabolic age $(p=0.01)$, lean body mass $(p<0.001)$ and basal metabolic rate was significantly higher in Group 2. The highest positive correlation with EAT value was found with visceral fat ratio $(r=0.600, p<0.001)$.

Conclusions: Malnutrition is a frequent problem in the HD population. We found low EAT in patients with malnutrition. As a result, we think that EAT can be used as a risk factor for KVC in patients without malnutrition.

Keywords: Hemodialysis; Epicardial adipose tissue; Malnutrition; Body composition; Cardiovascular risk factor; Chronic kidney disease; Echocardiography

\section{Introduction}

Patients with ESRD receiving HD treatment have been 2075\% PEM [1,2]. This can be result from factors such as uremia, inflammation, multiple drug use, physical barriers, dietary restrictions, gastrointestinal system disorders, metabolic acidosis or HD therapy itself [2]. In this population it is important to assess nutritional status and body composition correctly. Because protein-energy malnutrition is the strongest risk factor for morbidity and mortality $[1,3,4]$.

Cardiovascular disorders (CVD) are the most common cause of morbidity and mortality in HD patients [5]. In this population, traditional CVD contribute to poor prognosis, as well as nontraditional risk factors such as malnutrition, hypervolemia and uremia [6]. EAT has been noted in recent years as a factor associated with coronary artery disease (CAD). EAT is a metabolically active visceral fat reservoir with more inflammatory activity than the subcutaneous fat or visceral adipose tissue [7]. It has been accepted as a new risk factor for CVD in non-uremic patients. But, the role of EAT in HD patients is still unknown [8].

To assess the PEM; clinical evaluation, weight loss narrative, serum albumin, creatinine, lipid levels, BMI and anthropometric measurements are used [9]. However, these methods may be misleading in some cases and it may be useful to evaluate classical methods together with methods such as MNA, dual-energy X-ray absorptiometry (DEXA) or bioelectric impedance analysis (BIA). MNA is an important method for evaluating PEM and has been recommended by the European Association of Parenteral and Enteral Nutrition [10].
Our aim was to compare EAT in HD patients with and without malnutrition. Our second objective was to determine whether the role of EAT in HD patients can be used to determine cardiovascular risk factor in HD patients with malnutrition.

\section{Materials and Methods}

Fifty-six patients were included in the study who were receiving HD therapy for ESRD. The study was approved by the local ethical committee (date: 25/10/2017, decision no: 227199). Written consent was taken from all of the patients included in the study. Study popülation were selected from patients who were eligible of consciousness to respond the questionnaire. Those who were 18 years or older age, who had been on HD therapy for at least 3 months and had not received psychiatric treatment in the last 3 months, were included in the study. Malignancy, infection and tuberculosis, and those who did not want to participate in the study were excluded from the study. All patients underwent bicarbonate hemodialysis. The mean blood flow rate was maintained at $280-350 \mathrm{~mL} /$ $\mathrm{min}$ and the $\mathrm{Kt} / \mathrm{V}$ value of all patients was $>1.2$ calculated.

*Corresponding author: Demir M, Division of Nephrology, Firat University School of Medicine, Elazig, Turkey, Tel: +90 543767 2994; E-mail: drmusdem@gmail.com

Received: July 29, 2019; Accepted: November 04, 2019; Published: November 08, 2019

Citation: Demir M, Canpolat O, Cerci AD, Dogukan A (2019) The Role of Epicardial Fat Tissue at Cardiovascular Risk Assessment in Hemodialysis Patients with Nutritional Disorder. J Nephrol Ther 9: 329.

Copyright: $\odot 2019$ Demir M, et al. This is an open-access article distributed under the terms of the Creative Commons Attribution License, which permits unrestricted use, distribution, and reproduction in any medium, provided the original author and source are credited. 
MNA questionnaire was applied to determine the nutritional status of the patients. Standard MNA consists of four components and 18 questions; Includes acute disease history, neuropsychological problems, anthropometric measurements and BMI as well as decreased food intake for 3 months, weight loss during the last 3 months, physical activity, and psychological stress (10). According to the MAN scores patients divided to two groups; PEM+PEM risk group (group 1, $\mathrm{n}=25$, score $<24$ ) and those with good nutritional status (group 2, n=31, score $\geq 24$ ).

Blood serum albumin, total cholesterol, triglyceride, CRP, calcium phosphor, hemoglobin and creatinine values and other parameters were evaluated retrospectively.

Tanita SC 330, a body composition analyzer, was used to evaluate the body composition of the patients. Measurements were made 20 minutes after the hemodialysis treatment in accordance with the manufacturer's recommendation and patients were allowed to stand with the bare feet on the device. Parameters such as lean body mass, fat mass, fat mass percentage (fat mass/weight), weight, visceral fat ratio, body mass index, basal metabolic rate, muscle mass and lean body mass were evaluated.

Transthoracic ECO was also performed by the cardiologist. Cardiac functions was evaluated with epicardial fat thickness, heart valve calcification, LVED, LVES.

\section{Statistical Analysis}

Data obtained in the study were statistically evaluated using the SPSS 20.0 (Statistical Package for Social Sciences for Windows SSPS Inc, Chicago, IL, USA) program. Descriptive statistical data were expressed as mean \pm standard deviation or median (min-max). The $t$ test was used to compare numerical data among the groups, and the Chi square analysis was used to compare categorical data. Pearson correlation test was used to determine the relationship between numerical variables. The results were stated at $95 \%$ confidence interval, $\mathrm{p}<0.05$ significance level.

\section{Results}

Of the 56 patients included in the study, 31 were male and 25 were female. The mean age of study popülasion was $55 \pm 14$.17. In group 2 , mean age $(\mathrm{p}=0.049)$ and male/female ratio $(\mathrm{p}=0.009)$ were significantly higher than group 1.

There was a significant difference between the groups in terms of EAT value ( $\mathrm{p}=0.032)$. ET value was higher in Group 2 than in Group 1. When serum $P(p=0.01)$ and CAXP $(p=0.02)$ values were compared between the two groups, both parameters were significantly higher in Group 2. In addition, fat mass $(\mathrm{p}=0.011)$, visceral fat percentage $(\mathrm{P}<0.001)$, muscle mass $(\mathrm{p}<0.001)$, metabolic age $(\mathrm{p}=0.01)$ and lean body mass $(\mathrm{p}<0.001)$ were significantly higher in group 2 . As expected, the BMI and weight was high in the Well-Nourished Group. Comparisons of parameters between groups are summarized in Table 1.

EAT value was correlated with some parameters and the highest positive correlation was found with visceral fat ratio $(r=0.600$, $\mathrm{p}<0.001$ ) (Figure 1). There was also a moderate correlation with age $(\mathrm{r}=0.594, \mathrm{p}<0.001)$ and metabolic age $(\mathrm{r}=0.501, \mathrm{p}<0.001)$ (Figure 2$)$ Furthermore, low correlation was found with $\mathrm{CRP}(\mathrm{R}=0.408, \mathrm{p}=0.002)$, fat mass ( $r=0.388, p=0.003), B M I(r=0.398, p=0.002)$, FAT\% $(r=0.305$, $\mathrm{p}=0.026)$.

\section{Discussion}

Proper evaluation of nutritional status in dialysis patients is important. Malnutrition causes an increase in the hospitalization rate and a delay in the healing of the infection [3]. It also contributes

\begin{tabular}{|c|c|c|c|c|}
\hline & PEM+PEM Risk Group (Grup 1) & Well-Nourished Group (Grup 2) & Total & $P$ value \\
\hline Age (year) & $50.88 \pm 15.9$ & $58.35 \pm 11.8$ & $55 \pm 14.17$ & 0.049 \\
\hline Gender (M/F) & $9 / 16$ & $22 / 9$ & $31 / 25$ & 0.009 \\
\hline Weight (kilogram) & $57 \pm 9.81$ & $72 \pm 10.59$ & $65.74 \pm 12.76$ & $<0.001$ \\
\hline Length $(\mathrm{cm})$ & $158.0 .4 \pm 10.80$ & $163.58 \pm 11.13$ & $161.10 \pm 11.23$ & 0.066 \\
\hline $\mathrm{BMI}\left(\mathrm{kg} / \mathrm{m}^{2}\right)$ & $22.92 \pm 3.96$ & $27.46 \pm 5.19$ & $25.44 \pm 5.17$ & 0.001 \\
\hline $\mathrm{EAT}(\mathrm{mm})$ & $5.76 \pm 1.56$ & $6.68 \pm 1.54$ & $6.27 \pm 1.6$ & 0.032 \\
\hline EF & $60(55-65)$ & $60(55-65)$ & $60(55-65)$ & 0.073 \\
\hline LVED & $45.27 \pm 13.14$ & $45.68 \pm 5.82$ & $45.51 \pm 9.45$ & 0.880 \\
\hline LVES & $25.14 \pm 5.46$ & $28.19 \pm 4.22$ & $26.92 \pm 4.64$ & 0.026 \\
\hline Visceral fat ratio (\%) & $6.48 \pm 4.01$ & $11.16 \pm 4.62$ & $9.07 \pm 4.92$ & $<0.001$ \\
\hline Total body fat ratio (\%) & $24.13 \pm 9.92$ & $27.28 \pm 11.21$ & $25.87 \pm 10.68$ & 0.276 \\
\hline Lean body mass $(\mathrm{kg})$ & $42.96 \pm 8.05$ & $52.33 \pm 7.7$ & $48.14 \pm 9.13$ & $<0.001$ \\
\hline Metabolic Age (year) & $40.84 \pm 16.59$ & $53.23 \pm 17.88$ & $47.70 \pm 18.25$ & 0.01 \\
\hline Basal metabolic speed (kcal) & $1272 \pm 199.55$ & $1533 \pm 200.12$ & $1416 \pm 237.57$ & $<0.001$ \\
\hline $\mathrm{Hb}(\mathrm{g} / \mathrm{dl})$ & $10.9(8.6-16.37)$ & $11.5(7.5-14.6)$ & $11.0 .5(7.50-16.37)$ & 0.225 \\
\hline Total cholesterol (mg/dl) & $193.56 \pm 70.08$ & $178.68 \pm 36.52$ & $185.32 \pm 54.09$ & 0.310 \\
\hline Triglyceride (mg/dl) & $201.32 \pm 70.62$ & $189.26 \pm 92.55$ & $194.64 \pm 82.97$ & 0.593 \\
\hline CRP (mg/L) & $7.2(3-29.70)$ & $6(3-88.5)$ & $5.75(3-88.5)$ & 0.373 \\
\hline Albumin (g/dl) & $3.7 \pm 0.67$ & $3.8 \pm 0.35$ & $3.8 \pm 0.52$ & 0.193 \\
\hline Creatinine (mg/dl) & $6.11 \pm 1.43$ & $7.02 \pm 1.78$ & $6.63 \pm 1.68$ & 0.051 \\
\hline Calcium (mg/dl) & $8.85 \pm 0.69$ & $8.89 \pm 0.73$ & $8.88 \pm 0.71$ & 0.911 \\
\hline Phosphor (mg/dl) & $4.17 \pm 0.91$ & $5.05 \pm 1.42$ & $4.66 \pm 1.28$ & 0.010 \\
\hline CAXP (mg/dl) & $37.07 \pm 9$ & $44.41 \pm 13.18$ & $41.13 \pm 11.99$ & 0.021 \\
\hline Uric acid (mg/dl) & $5.86 \pm 1.14$ & $6.80 \pm 1.63$ & $6.38 \pm 1.49$ & 0.018 \\
\hline
\end{tabular}

PEM: Protein-Energy Malnutrition; CRP: C-Reactive Protein; BMI: body mass index; Hb: Hemoglobin; CAXP: Calcium X Phosphor; EAT: Epicardial Adipose Tissue; EF: Ejection Fraction; LVED: Left Ventricular End-Diastolic; LVES: Left Ventricular End-Systolic.

Table 1: Comparison of demographic clinical, biochemical, and nutritional parameters of the groups. 


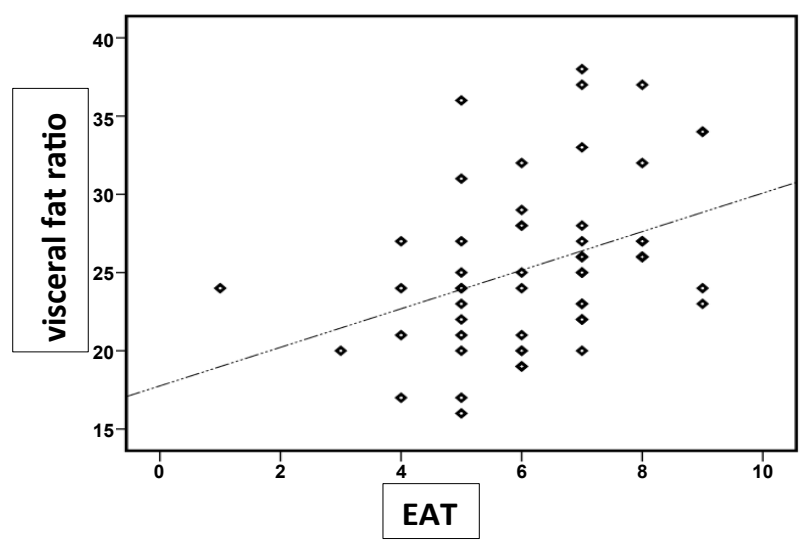

Figure 1: The relationship between epicardial adipose tissue (EAT) and visceral fat ratio.

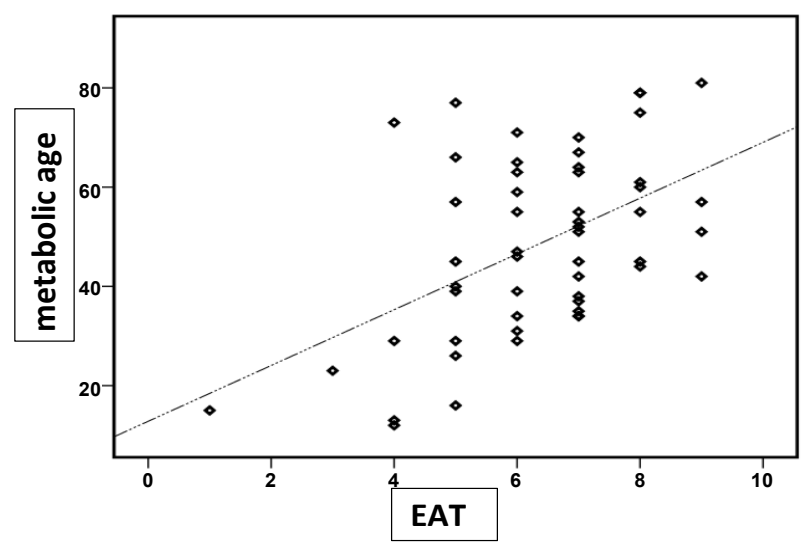

Figure 2: The relationship between epicardial adipose tissue (EAT) and metabolic age.

to high morbidity and mortality [11]. Weight loss narrative, serum albumin, creatinine and lipid profile, biochemical markers, BMI and anthropometric measurements can be used to assess the PEM. In addition to these conventional methods, MNA, DEXA or BIA can also be used for nutritional evaluation [10]. In this study, the BIA was used together with MNA in the evaluation of malnutrition.

Biochemical parameters are known to be complementary to information obtained by other nutritional evaluation methods such as clinical, diet diary and anthropometry in evaluation of nutrition [5]. Fiedler et al. [9] demonstrated that malnutrition identified by clinical assessment score (MIS, NRS, SGA) and biochemical markers (ALB, PRA, transferrin) correlated with mortality in HD patients with serum CRP levels. There was a significant difference in biochemical parameters between the two groups in terms of phosphorus, CAXP and uric acid levels in our study. Albumin levels were not different. This can be explained by two ways. First, low levels of serum albumin usually occur during the late stages of malnutrition [11]. However, MNA identifies patients with short-term nutritional problems associated with reduced food intake within 1-2 weeks [10]. Albumin level may be affected by longer nutritional deficiencies due to half-life. The second reason is that hypoalbuminemia does not always mean malnutrition induced by low protein intake, because frequent overhydration in chronic HD patients and hemodiluation can also cause hypoalbuminemia [12].
In addition, albumin levels may be affected by albumin synthesis inhibition, passing to dialysate during hemodiyaliz, changes between intravascular and extravascular compartments, and most importantly chronic inflammatory conditions [10].

Serum creatinine is also considered nutritional indicators and are reported among prognostic factors in HD patients [13]. Creatinine level is influenced by residual renal function, size of the patient's muscle mass, advanced age, male sex, and meat consumption in large quantities [10]. In our results, there was a difference in creatinine level between the groups but this difference was not statistically significant. This result suggests that the evaluation of malnutrition alone with biochemical results will lead to a mistake. due to similar concerns Fiedler et al. recommended that using at least two clinical nutrition scoring (eg laboratory methods, BIA, BMI...) in the evaluation of nutrition [9].

BIA is another method of analysis that used in assessing nutritional status in chronic kidney disease (CKD). In our study, we choosed to the MAN with proven reliability in assessing malnutrition and also used the BIA. Determination of nutritional status by body composition analysis in CKD patients [5], provides important clinical information necessary to prescribe and monitor appropriate nutritional therapy [1]. In addition, it allows to evaluating volume status and to perform appropriate ultrafiltration. [5]. Yue et al. reported that they had measured the body fat percentage by the BIA method in patients and found that only 1 of $84(1.2 \%)$ patients had body fat percentage below normal, while the other 83 patients found that they were within the reference range. Interestingly, the same patient group was evaluated by modified quantitative subjective assessment (MQSGA); In 55 of 84 (65.5\%) patients the MQSGA score was higher than 7. The percentage of malnourished patients identified by MQSGA and BIA in the mirror patient group was significantly different [11]. In our study, body fat and visceral fat ratio obtained with BIA were significantly lower in Group 1 than Group 2 patients. These results, contrary to the findings of Yue et al., suggests that use of the BIA in nutritional evaluation is as sensitive as other nutritional measurement methods. In an other study in which nutritional status was assessed by different methods in geriatric hemodialysis patients, the frequency of malnutrition in geriatric HD patients varied according to the method and sex [3].

EAT has been described as a risk factor for CVD in the non-üremic population [12-14]. Cardiovascular diseases are the most common cause of death in ESRD patients. It constitutes about $50 \%$ of deaths [12]. The increased incidence of CVD in HD patients has forced investigators to find new, potentially interchangeable risk factors in this patient population, apart from classical risk factors. One of these newly identified risk factors is the EAT surrounding the epicardial vessels [14]. The role of EAT in uremic patients is not clear. However, EFT in uremic patients has recently been investigated in various studies. EFT is also originated from splancnopleuric mesoderm, such as abdominal visceral fat tissue [8]. Metabolically, it is an active organ that produces many cytokines including TNF-a, IL-6, omentin, leptin, angiotensinogen PAI-1,which are mainly proinflammatory and proatherogenic [14]. EAT increases proportionally with body fat [7]. Doesch et al. reported that EAT was closely associated with visceral fat tissue but not with total fat mass in metabolic syndrome patients with normal renal function [15]. Erdur et al. found that EAT levels were significantly higher in HD patients than healthy populations [16]. Turkmen et al. found a correlation between EAT level and MIAC syndrome in ESRD patients. EAT was significantly increased when the number of MIAC components increased [17]. 
Citation: Demir M, Canpolat O, Cerci AD, Dogukan A (2019) The Role of Epicardial Fat Tissue at Cardiovascular Risk Assessment in Hemodialysis Patients with Nutritional Disorder. J Nephrol Ther 9: 338.

There are some limitations in our study. One of the most important limitations of our study was the cross-sectional design. It may affect cause-and-effect relationships. So for this reason long-term perspective analyzes are required.

\section{Conclusion}

In conclusion, Low EAT level in patients with malnutrition has led to questioning the role of EAT in assessing the risk of CVD in HD patients. Because malnutrition is a frequent problem in the HD population, it is important to know whether patients have malnutrition in order to be able to evaluate EAT as a cardiovascular risk factor in this patients group. As a result, we think that EAT can be used as a risk factor for KVC in patients without malnutrition.

\section{Conflict of Interest}

The authors declare that they have no conflict of interest.

\section{References}

1. Bross R, Chandramohan G, Kovesdy CP, Oreopoulos A, Noori N, et al. (2010) Comparing Body Composition Assessment Tests in Long-term Hemodialysis Patients. Am J Kidney Dis 55: 885-896.

2. Rimsevicius L, Gincaite A, Vicka V, Sukackiene D, Pavinic J, et al. (2016) Malnutrition Assessment in Hemodialysis Patients: Role of Bioelectrical Impedance Analysis Phase Angle. Journal of Renal Nutrition 26: 391-395.

3. Kaya T, Sipahi S, Karacaer C, Nalbant A, Varım C, et al. (2014) Evaluation of nutritional status with different methods in geriatric hemodialysis patients: impact of gender. Int Urol Nephrol 46: 2385-2391.

4. Piccoli A, Codognotto M, Piasentin P, Naso A (2014) Combined evaluation of nutrition and hydration in dialysis patients with bioelectrical impedance vector analysis (BIVA). Clinical Nutrition 33: 673-677.

5. Cuevas E, Rodriguez1 N, Martinez V, Carsi A, Alatriste M, et al. (2010) Body fluid volume and nutritional status in hemodialysis: vector bioelectric impedance analysis. Clin Nephrol 73: 300-308.

6. Antunes AA, Vannini FD, Silveira LVA, Barretti P, Martin LC, et al. (2013) Associations between bioelectrical impedance parameters and cardiovascular events in chronic dialysis patients. Int Urol Nephrol 45: 1397-1403.
7. Lee BC, Lee WJ, Lo SC, Hsu HC, Chien KL, et al. (2016) The ratio of epicardia to body fat improves the prediction of coronary artery disease beyond calcium and Framingham risk scores. Int J Cardiovasc Imaging 32: S117-127.

8. Atakan A, Macunluoglu B, Kaya Y, Arı E, Demır H, et al. (2014) Epicardial fat thickness is associated with impaired coronary flow reserve in hemodialysis patients. Hemodial Int 18: 62-69.

9. Fiedler R, Jehle PM, Osten B, Dorligschaw O, Girndt M (2009) Clinical nutrition scores are superior for the prognosis of hemodialysis patients compared to lab markers and bioelectrical impedance. Nephrol Dial Transplant 24: 3812-3817.

10. Erdogan E, Tutal E, Uyar ME, Bal Z, Demirci BG, et al. (2013) Reliability of Bioelectrical Impedance Analysis in the Evaluation of the Nutritional Status of Hemodialysis Patients - A Comparison With Mini Nutritional Assessment. Transplant Proc 45: 3485-3488.

11. Hou Y, Li X, Hong D, Zou H, Yang L, et al. (2012) Comparison of different assessments for evaluating malnutrition in Chinese patients with end-stage renal disease with maintenance hemodialysis. Nutr Res 32: 266-271.

12. Hyun SH, Choi JY, Cho JH, Park SH, Kim CD, et al. (2014) Assessment of Fluid and Nutritional Status Using Multifrequency Bioelectrical Impedance Analysis in Peritoneal Dialysis Patients. Blood Purif 37: 152-162.

13. Vannini FD, Antunes AA, Caramori JCT, Martin LC, Barretti P (2009) Associations between nutritional markers and inflammation in hemodialysis patients. Int Urol Nephrol 41:1003-1009.

14. Macunluoglu B, Atakan A, Ari E, Kaya Y, Kaspar C, et al. (2014) Epicardia fat tissue thickness is correlated with diminished levels of co-enzyme Q10, a major antioxidant molecule among hemodialysis patients. Clin Biochem 47 1231-1234.

15. Doesch C, Suselbeck T, Leweling H, Fluechter S, Haghi D, et al. (2010) Bioimpedance analysis parameters and epicardial adipose tissue assessed by c ardiac magnetic resonance Imaging in patients with heart failure. Obesity 18 : 2326-2332.

16. Erdur MF, Tonbul HZ, Ozbiner H, Ozcicek A, Ozcicek F, et al. (2013) The relationship between atherogenic index of plasma and epicardial adipose tissue in hemodialysis and peritoneal dialysis patients. Ren Fail 35: 1193-1198.

17. Turkmen K, Kayikcioglu H, Ozbek O, Solak Y, Kayrak M, et al. (2011) The Relationship between Epicardial Adipose Tissue and Malnutrition, Inflammation, Atherosclerosis/Calcification Syndrome in ESRD Patients. Clin J Am Soc Nephrol 6: 1920-1925. 